\title{
Integrating RNA-Seq with GWAS reveals novel insights into the molecular mechanism underpinning ketosis in cattle
}

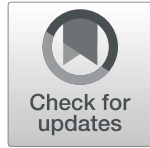

Ze Yan ${ }^{1}$, Hetian Huang ${ }^{1,2}$, Ellen Freebern ${ }^{3}$, Daniel J. A. Santos ${ }^{3}$, Dongmei Dai ${ }^{1}$, Jingfang $\mathrm{Si}^{1}$, Chong Ma ${ }^{4}$, Jie Cao ${ }^{4}$, Gang Guo ${ }^{5}$, George E. Liư ${ }^{6}$ Li Ma ${ }^{3^{*}}$, Lingzhao Fang ${ }^{7^{*}}$ (D) and Yi Zhang ${ }^{1^{*}}$

\begin{abstract}
Background: Ketosis is a common metabolic disease during the transition period in dairy cattle, resulting in longterm economic loss to the dairy industry worldwide. While genetic selection of resistance to ketosis has been adopted by many countries, the genetic and biological basis underlying ketosis is poorly understood.

Results: We collected a total of 24 blood samples from 12 Holstein cows, including 4 healthy and 8 ketosisdiagnosed ones, before ( 2 weeks) and after ( 5 days) calving, respectively. We then generated RNA-Sequencing (RNASeq) data and seven blood biochemical indicators (bio-indicators) from leukocytes and plasma in each of these samples, respectively. By employing a weighted gene co-expression network analysis (WGCNA), we detected that 4 out of 16 gene-modules, which were significantly engaged in lipid metabolism and immune responses, were transcriptionally $(F D R<0.05)$ correlated with postpartum ketosis and several bio-indicators (e.g., high-density lipoprotein and low-density lipoprotein). By conducting genome-wide association signal (GWAS) enrichment analysis among six common health traits (ketosis, mastitis, displaced abomasum, metritis, hypocalcemia and livability), we found that 4 out of 16 modules were genetically (FDR $<0.05)$ associated with ketosis, among which three were correlated with postpartum ketosis based on WGCNA. We further identified five candidate genes for ketosis, including GRINA, MAFI, MAFA, C14H8orf82 and RECQL4. Our phenome-wide association analysis (Phe-WAS) demonstrated that human orthologues of these candidate genes were also significantly associated with many metabolic, endocrine, and immune traits in humans. For instance, MAFA, which is involved in insulin secretion, glucose response, and transcriptional regulation, showed a significantly higher association with metabolic and endocrine traits compared to other types of traits in humans.
\end{abstract}

(Continued on next page)

\footnotetext{
* Correspondence: lima@umd.edu; Lingzhao.fang@igmm.ed.ac.uk;

yizhang@cau.edu.cn

${ }^{3}$ Department of Animal and Avian Sciences, University of Maryland, College

Park, MD 20742, USA

${ }^{7}$ MRC Human Genetics Unit at the Institute of Genetics and Molecular

Medicine, University of Edinburgh, Edinburgh EH4 2XU, UK

${ }^{1}$ National Engineering Laboratory for Animal Breeding, Key Laboratory of

Animal Genetics, Breeding and Reproduction of Ministry of Agriculture and

Rural Affairs, College of Animal Science and Technology, China Agricultural

University, Beijing 100193, China

Full list of author information is available at the end of the article
}

(c) The Author(s). 2020 Open Access This article is licensed under a Creative Commons Attribution 4.0 International License, which permits use, sharing, adaptation, distribution and reproduction in any medium or format, as long as you give appropriate credit to the original author(s) and the source, provide a link to the Creative Commons licence, and indicate if changes were made. The images or other third party material in this article are included in the article's Creative Commons licence, unless indicated otherwise in a credit line to the material. If material is not included in the article's Creative Commons licence and your intended use is not permitted by statutory regulation or exceeds the permitted use, you will need to obtain permission directly from the copyright holder. To view a copy of this licence, visit http://creativecommons.org/licenses/by/4.0/ The Creative Commons Public Domain Dedication waiver (http://creativecommons.org/publicdomain/zero/1.0/) applies to the data made available in this article, unless otherwise stated in a credit line to the data. 
(Continued from previous page)

Conclusions: In summary, our study provides novel insights into the molecular mechanism underlying ketosis in cattle, and highlights that an integrative analysis of omics data and cross-species mapping are promising for illustrating the genetic architecture underpinning complex traits.

Keywords: GWAS, Holstein, Ketosis, RNA-Seq, Phe-WAS, WGCNA

\section{Background}

The transition period, known as 3 weeks pre- until 3 weeks post-calving, is a critical time for dairy cows since many metabolic and infectious diseases occur due to dramatic physiological challenges faced by cows (e.g., the negative energy balance, NEB) [1]. Ketosis is one of the most important metabolic disorders during transition period. It is often caused due to the severe imbalance between energy demands (e.g., high milk yield) and energy intake. The incidence of ketosis is as high as $15-30 \%$ in the dairy industry, and cows with high milk yield predispose to ketosis [2], leading to huge economic losses worldwide. For instance, each case of ketosis costs \$ 77.00-180.91 and $¥ 3200$ in the U.S. [3] and China [4] Holstein populations, respectively. Ketosis is usually clinically diagnosed by a concentration of $\beta$-hydroxybutyrate (BHBA) in plasma greater than $1.4 \mathrm{mmol} / \mathrm{L}$ [5-8]. Animals with ketosis are more susceptible to other transition-relevant diseases (e.g., displaced abomasum, DSAB; mastitis, MAST), which together have negative impacts on the performance of production (e.g., reduced milk yield) and reproduction (e.g., infertility) [3, 9].

Ketosis is a complex trait controlled by both genetic and environmental factors, with the estimated heritability ranging from 0.01 to 0.16 [10-13]. Our previous large-scale $(n \approx 10 \mathrm{~K}$ bulls $)$ genome-wide association study (GWAS) of ketosis (the estimated heritability was 0.012) detected only a few significant loci on Bos Taurus autosome (BTA) 14 and BTA16 in Holstein cattle, which together explained a small proportion of its entire genetic variance [10]. This finding strongly suggests a highly polygenetic architecture underlying ketosis. Previous studies proposed that genetic variants of complex traits are enriched in genes with similar biological functions (e.g., Gene Ontology terms) [14-18]. For instance, McCabe et al. (2012) previously demonstrated that differentially expressed genes (DEGs) induced by different energy conditions (i.e., mild NEB and severe NEB) were significantly engaged in fatty acid metabolism and steroid hormone biosynthesis [19]. Therefore, it is of great interest to detect genes that function together during ketosis by using RNA sequencing (RNA-Seq), and then test whether genetic variants of ketosis are enriched in these genes.

In this study (Fig. 1), to explore the genetic architecture underlying ketosis, we generated RNA-Seq of blood leukocytes and biochemical indicators (bio-indicators) of plasma from both healthy and ketosis-diagnosed cows. We then integrated RNA-Seq with large-scale GWAS $(n \approx 10 \mathrm{~K})$ of ketosis and other five health traits, including livability, DSAB, hypocalcemia (CALC), MAST and metritis (METR). We further validated our ketosiscandidate genes using the phenome-wide association analysis (Phe-WAS) based on human databases.

\section{Results}

Summary of RNA-Seq data

In total, we generated 24 RNA-seq data from 12 Holstein cows, including 4 healthy and 8 ketosis-diagnosed ones, before ( 2 weeks) and after ( 5 days) calving, respectively. After the quality control of raw RNA-Seq data (in Methods), we obtained a total of 1,286,805,582 clean paired-end reads. By aligning clean data to the cattle reference genome (UMD3.1.1), we obtained an averaged mapping rate of $94.76 \%$ (ranging from 93.86 to $95.73 \%$ ) among all of the 24 samples. We summarized the detailed mapping information for all samples in Additional file 1: Table S1. Ultimately, we observed an average of 13,031 genes (ranging from 12,683 to 13,248 ) that were expressed (transcripts per kilobase million, TPM >1) across 24 samples. We then kept 13,600 genes that were expressed in at least one sample and had median absolute deviation (MAD) greater than 0.01 (the top $75 \%$ of $\mathrm{MAD}$ ) for the subsequent analyses.

\section{Gene co-expression modules associated with ketosis and biochemical indicators}

By employing a weighted correlation network analysis (WGCNA) on all 24 blood leukocytes RNA-Seq data, we detected 16 gene modules (15 co-expression modules and 1 module with the remaining uncorrelated genes), among which the number of genes ranged from 147 to 3178 (Fig. 2a). We then calculated associations of each module with four physiological states (i.e., pre-partum healthy, post-partum healthy, pre-partum ketosis, and postpartum ketosis) and seven blood bio-indicators, including BHBA, total cholesterol (TC), total triglyceride (TG), highdensity lipoprotein (HDL), low-density lipoprotein (LDL), calcium (Ca), and insulin (INS) (Additional file 2: Table S2), respectively. Interestingly, we found that three modules, Royalblue, Black, and Darkorange, were significantly (FDR < 0.05 ) and specifically associated with post-partum ketosis (Fig. 2b). We also found another module, Midnightblue, 


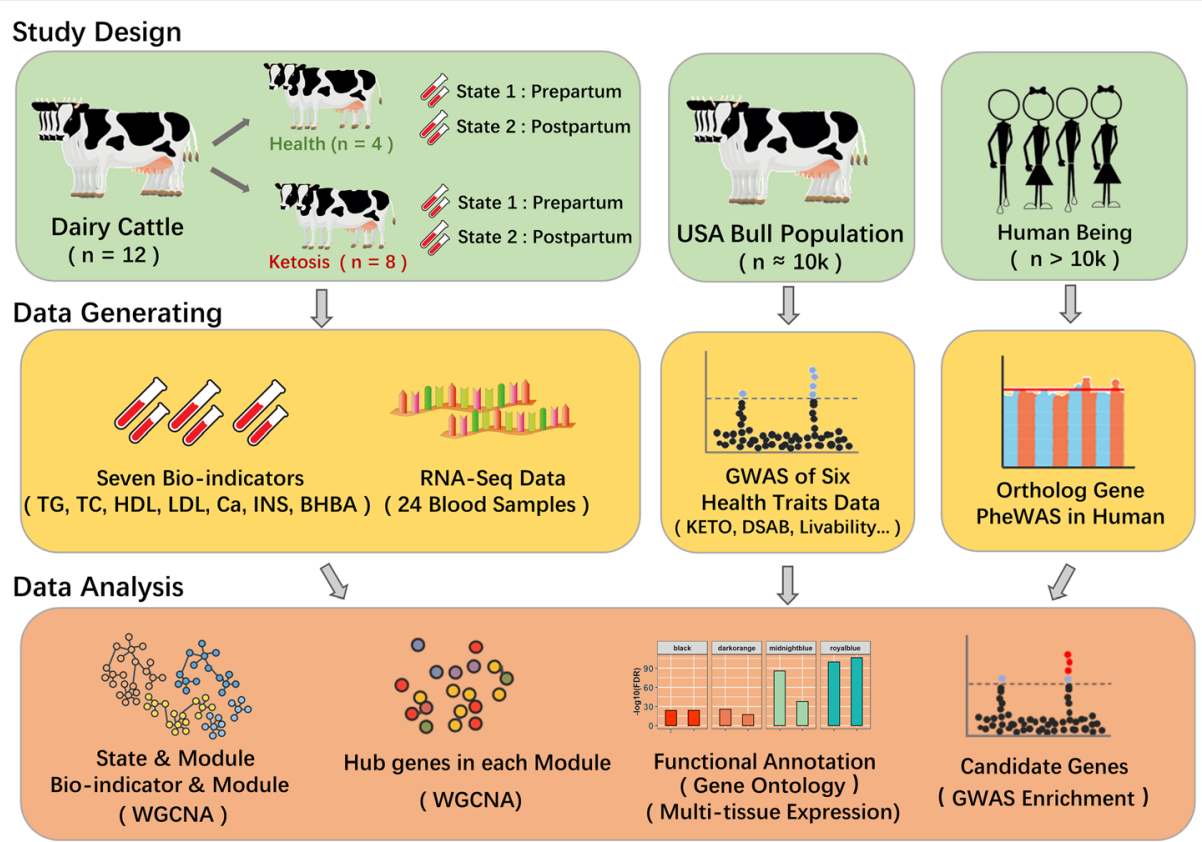

Fig. 1 Global framework of the study. The green box (left) represents the experimental design of RNA-Seq study. We selected 12 Holstein cows, among which eight were ketosis (BHBA>1.4 mmol/L), and the remaining four were healthy $(B H B A<1.4 \mathrm{mmol} / \mathrm{L})$. We collected the whole blood samples from each individual before ( 2 weeks; prepartum) and after (5 days; postpartum) calving, respectively. The other green boxes (right) demonstrate materials used in genome-wide association studies (GWAS) in cattle and phenome-wide association studies (Phe-WAS) in human. The orange boxes are for data generating, including RNA-Seq and seven blood bio-indicators data from all 24 blood samples, GWAS of six traits (livability; ketosis, KETO; displaced abomasum, DSAB; hypocalcemia, CALC; mastitis, MAST; metritis, METR) and Phe-WAS data (https://atlas.ctglab. $\mathrm{nl} /$ ). The brown box shows major bioinformatics and statistical analyses involved in the study

which tended to be $(P=0.008$, FDR $=0.10)$ associated with post-partum ketosis. Gene Ontology enrichment analysis showed that genes in the Royalblue module were significantly $(\mathrm{FDR}<0.05)$ involved in the microtubule-based and macromolecule biosynthetic processes, while genes in the remaining three modules were significantly engaged in immune responses (Fig. 2c, Additional file 3: Table S3). The tissue/cell type-enrichment analysis also confirmed that genes in Royalblue were significantly $(\mathrm{FDR}<0.05)$ enriched for gene with specific expression in digestive and immune systems (e.g., diaphragm and gall bladder), while genes in the remaining three modules were significantly enriched for genes with specific expression in the blood and immune system (Fig. 2d, Additional file 4: Table S4). In addition, we noticed that a module, Lightcyan, appeared to be $(\mathrm{FDR}<0.1)$ associated with pre-partum ketosis. Genes in this module were significantly engaged in the nervous system (Additional file 3: Table S3), which might reflect the cross-talk between the nervous system and digestive/immune systems (i.e., the so-called gut-brain axis) [20-23].

We further explored associations of modules with seven plasma bio-indicators (Fig. 2b). As expected, we found that four post-partum ketosis-associated modules were associated with BHBA (FDR $<0.1)$. We also observed that two modules, Darkorange and Midnightblue, were associated with HDL, while Steelblue and Skyblue modules were associated with LDL and INS, respectively. The pre-partum ketosis-associated module, Lightcyan, tended to be $(P=0.02$, FDR $=0.13$ ) associated with INS (Fig. $2 b$ ). We detected hubgenes in each of these modules (Additional file 5: Table S5). For instance, we found that expression levels of gene C14H8orf82 (belonging to Midnightblue) and ACSS1 (Darkorange) were significantly and positively correlated with HDL among 24 samples, while EPB2 (Steelblue) and PLK1 (Lightcyan) were significantly and negatively correlated with LDL and INS, respectively (Fig. 3a). Furthermore, we observed distinct expression patterns of these genes in the post-partum ketosis group compared to others (Fig. 3b). For instance, C14H8orf82 and ACSS1 had lower expression levels in the post-partum ketosis group than in others, leading to a lower HDL level. In contrast, EPB2 and PLK1 exhibited higher expression levels in the post-partum ketosis group, resulting in lower levels of LDL and INS, respectively. The protein-protein interaction analysis also showed that EPB2 and PLK1 interacted with many genes within the corresponding modules, indicating their central regulatory roles in these modules (Fig. 3c).

\section{Gene co-expression modules enriched with GWAS signals of health traits}

To investigate whether gene co-expression modules were enriched with GWAS signals of ketosis and other 


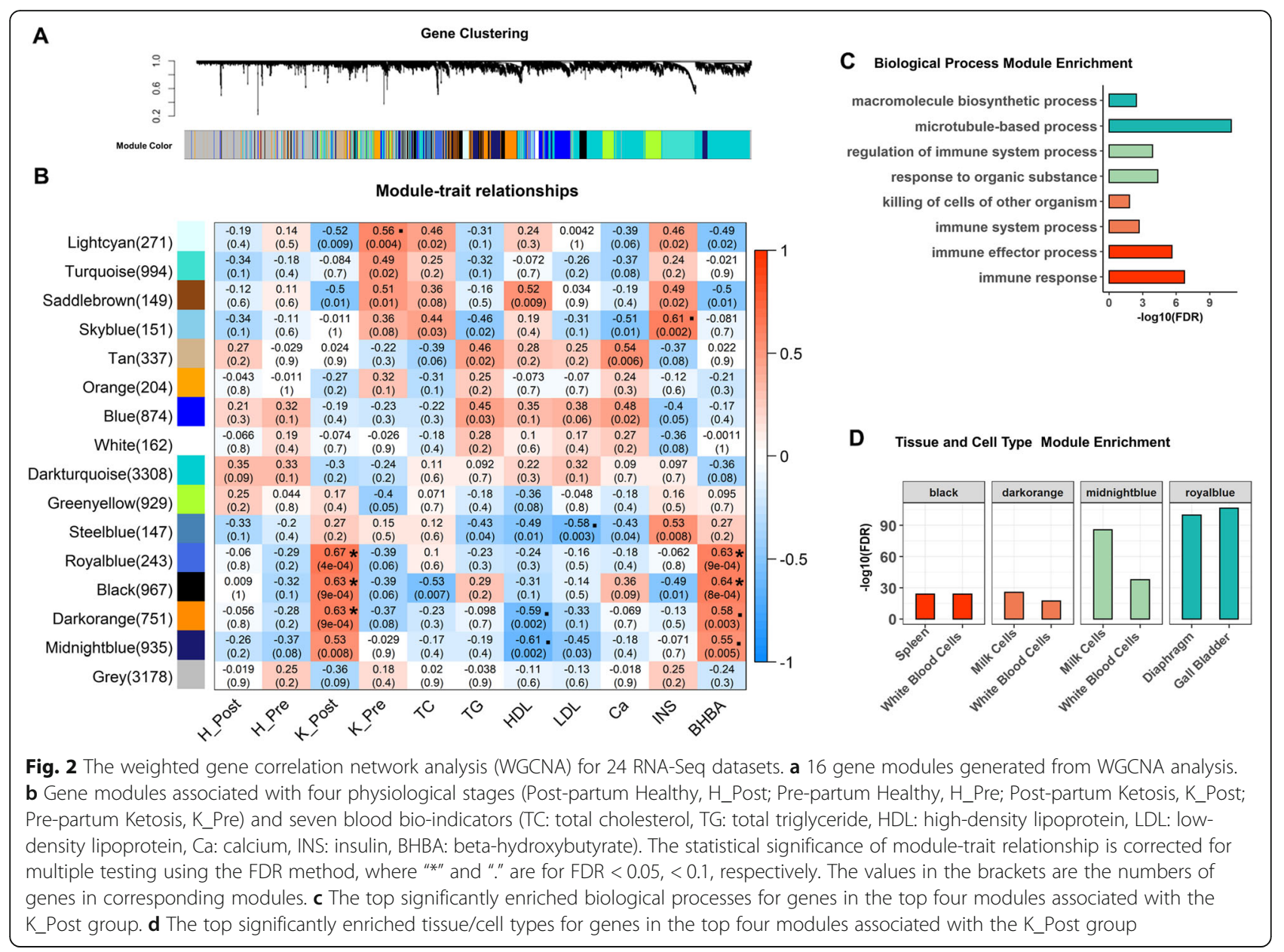

health traits, we applied GWAS enrichment analysis for all 16 gene modules across six health traits. As shown in Fig. 4a, several gene modules were significantly (FDR < 0.05 ) enriched with GWAS signals of these traits, among which ketosis clustered together with DSAB, in line with that both of them are metabolic disorders. We found that four modules, Royalblue, Darkorange, Midnightblue and Orange, were significantly enriched for GWAS signals of ketosis (Fig. 4a). Of note were Royalblue, Darkorange and Midnightblue, whose expression levels were significantly correlated with post-partum ketosis as well (Fig. 2b). By correlating GWAS enrichments of ketosis and module-trait associations from WGCNA across all 16 modules, we only observed a significant correlation $(r=0.60, P=0.014)$ for post-partum ketosis rather than other status (Fig. 4b; Additional file 6: Figure S1). This suggests that transcriptomic alterations induced by post-partum ketosis were biologically and genetically associated with GWAS ketosis. We further detected five candidate genes for ketosis, namely MAFA, C14H8orf82, MAF1, GRINA and RECQL4, within the four significant modules (Table 1). These genes were located within the top QTL of ketosis on BTA14 (Fig. 4c) [10]. Furthermore, we found that these five candidate genes were also associated $(P<0.05)$ with DSAB and livability (Fig. $4 \mathrm{~d})$, providing evidence that they might play polytrophic effects in multiple metabolic disorders.

\section{Phenome-wide association analysis (Phe-WAS) for ketosis candidate genes in humans}

In order to investigate whether candidate genes of cattle ketosis function similarly in humans, we first conducted a homology alignment analysis of these genes. Our results demonstrated that sequences of all five candidate genes were highly conserved (>80\%) among mammals (Fig. 5a left). We took one gene (i.e., MAF BZIP Transcription Factor A - MAFA) as an example to show its sequence conservations among seven mammalian species compared with cattle (Fig. 5a right). Then, we conducted Phe-WAS analysis for human orthologues of these candidate genes across 3302 human phenotypes (https://atlas.ctglab.nl/). We found that these genes were significantly associated (FDR $<0.05)$ with many metabolic traits and other health-relevant traits in humans, such as endocrine and immunological traits, suggesting their conserved roles in the regulation of metabolism 


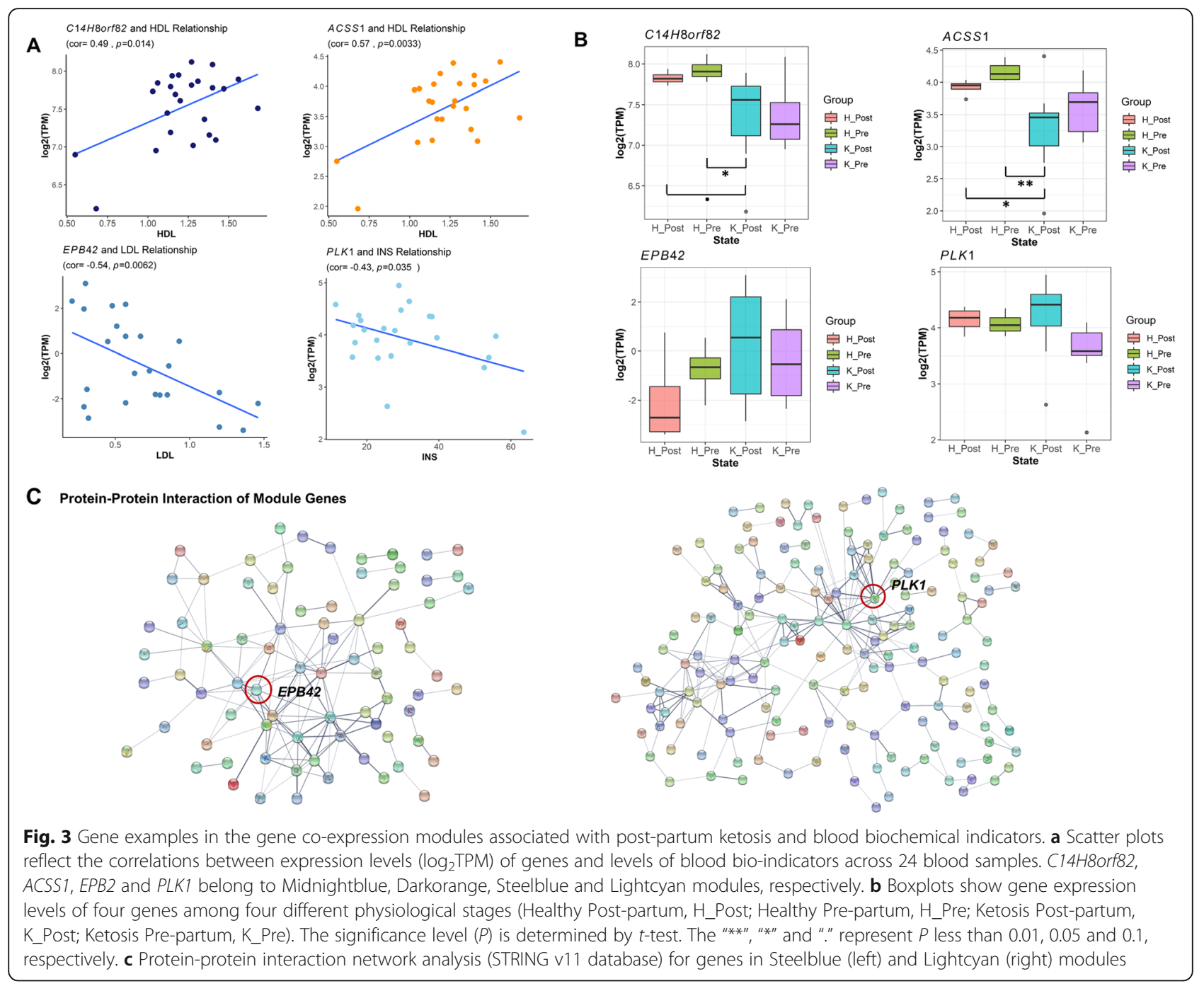

and potential pleiotropic effects on many health traits in mammals (Fig. 5b and c; Additional file 7: Table S6). We first took $M A F A$ as an example in Fig. 5b. Compared to other types of traits, MAFA showed a significantly higher association with metabolic and endocrine traits (e.g., Body fat percentage, $F D R=2.64 \mathrm{e}-05$; Type 2 Diabetes, FDR $=1.9 \mathrm{e}-03$ ). In addition, we showed Phe-WAS results for the remaining four candidate genes in Fig. 5c, namely MAF1, RECQL4, GRINA and C14H8orf82. MAF1 showed a significantly higher association with immunological traits (e.g., Platelet distribution width, FDR $=1.23 \mathrm{e}-09$ ) compared to other traits. It was also significantly associated with many endocrine traits (e.g., Insulin sensitivity index, FDR = 0.042; Type 2 Diabetes, FDR $=0.049)$. RECQL4 was significantly associated with many endocrine (e.g., Type 2 Diabetes, FDR $=4.53 \mathrm{e}-06$ ), immunological (e.g., Mean corpuscular hemoglobin concentration, FDR $=2.61 \mathrm{e}$ 11) and metabolic traits (e.g., Estimated glomerular filtration rate, $F D R=9.86 \mathrm{e}-06)$. It was reported to be associated with nucleic acid binding and annealing helicase activity [24, 25]. GRINA showed significant associations with metabolic (e.g., LDL cholesterol metabolism, FDR = 1.83e-07), immunological (e.g., Platelet distribution width, $\mathrm{FDR}=1.22 \mathrm{e}-22$ ) and cardiovascular traits (e.g., Coronary artery disease and low-density lipoprotein cholesterol, FDR $=1.01 \mathrm{e}-06$ ), and serves to function in apoptotic regulation [26]. C14H8orf82 was also significantly associated with many metabolic (e.g., Cholesterol esters in large LDL, $F D R=0.032$; Estimated glomerular filtration rate, $F D R=7.8 \mathrm{e}-04)$, immunological (Mean corpuscular haemoglobin concentration, FDR = 5.83e-05) and endocrine traits (e.g., Type 2 Diabetes, $F D R=0.0041)$. Our results here demonstrated that ketosis candidate genes detected in cattle might provide novel insights into the molecular mechanism underlying similar complex traits in humans, such as metabolic, immunological and endocrine traits. In turn, our study also demonstrated the potential of cross-species meta-analysis to improve the productivity of the cattle industry. 


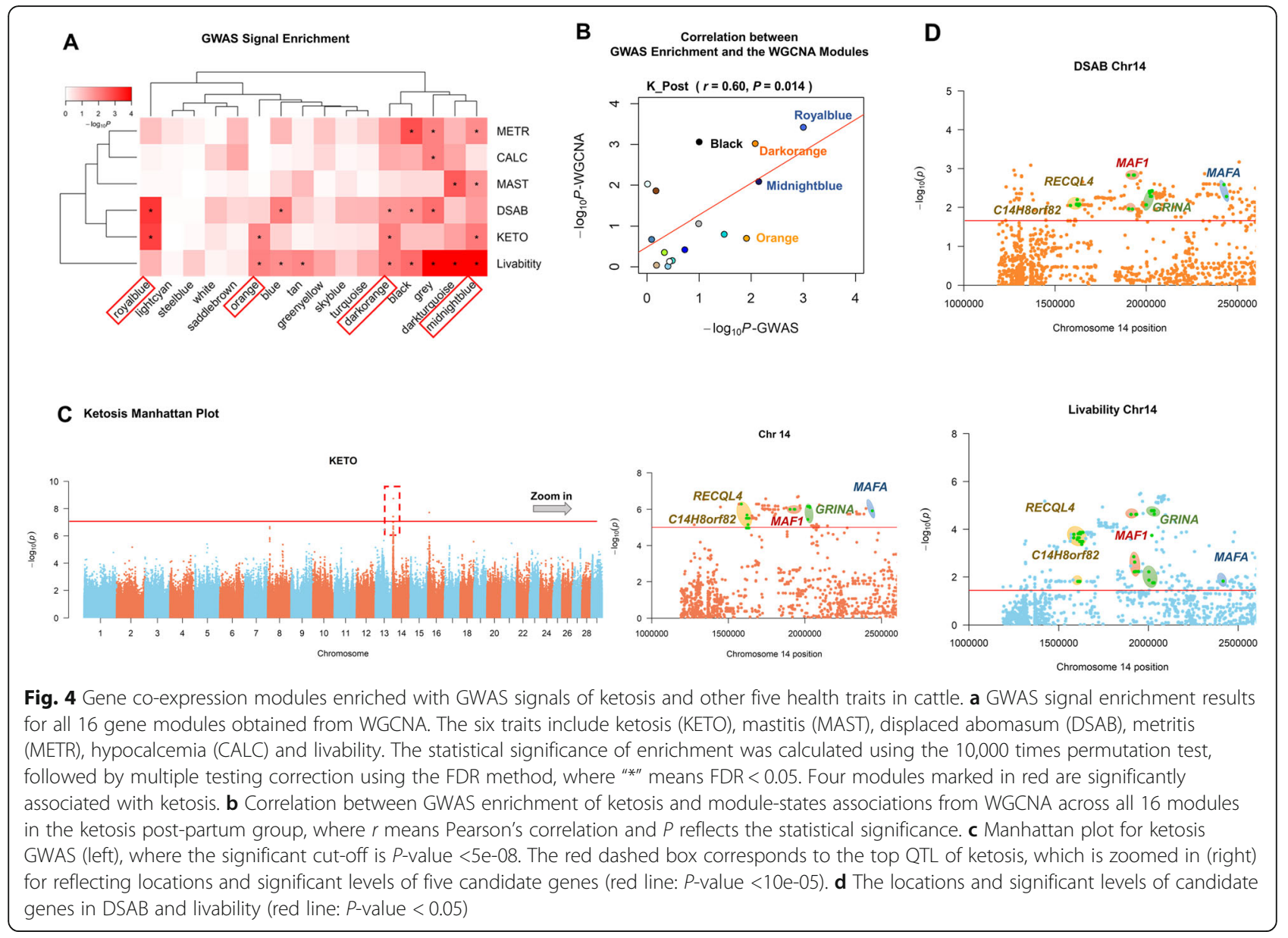

\section{Discussion}

To our best knowledge, this is the first study to explore the genetic and biological basis of ketosis in dairy cattle by systematically integrating RNA-Seq and large-scale GWAS data. Here, we applied the typical WGCNA strategy - single co-expression network analysis. By using samples of multiple status, a single co-expression network could identify common co-expression modules across status [27]. This analysis strategy has been widely used to detect genes that were associated with developmental stages of diseases, sex and tissues at a systemlevel [28-31]. For instance, a previous study detected candidate genes for High- and Sub-Fertile reproductive performance in beef cattle using WGCNA [32].
Compared to differential expression analyses at individual gene-level, WGCNA considers the relationship between altering genes as a whole, and reduces the multiple testing burden by focusing on tens of co-expression modules rather than thousands of individual genes. However, it is of note that the status/condition-specific co-expression modules may not be detected in the coexpression networks constructed from samples under multiple conditions, because the correlation signal of the condition-specific modules might be diluted by a lack of correlation in other conditions [27]. To identify modules unique to a specific condition, an alternative strategy, namely differential weighted gene co-expression network analysis (DWGCNA), could be used when sample size is

Table 1 Summary of five candidate genes for ketosis

\begin{tabular}{lllllll}
\hline Gene ID & Gene name & Chr. & Position of the top SNP (bp) & SNP effect & P-value & Module \\
\hline ENSBTAG00000000312 & GRINA & 14 & $2,016,987$ & 0.54 & $6.94 \mathrm{E}-07$ & Midnightblue \\
ENSBTAG00000012242 & MAF1 & 14 & $1,903,097$ & 0.53 & $1.00 \mathrm{E}-06$ & Midnightblue \\
ENSBTAG00000026344 & MAFA & 14 & $2,439,582$ & 0.47 & $1.21 \mathrm{E}-06$ & Midnightblue \\
ENSBTAG00000046031 & C14H8orf82 & 14 & $1,584,444$ & 0.55 & $5.17 \mathrm{E}-07$ & Midnightblue \\
ENSBTAG00000010276 & RECQL4 & 14 & $1,621,816$ & 0.51 & $3.10 \mathrm{E}-06$ & Royalblue \\
\hline
\end{tabular}




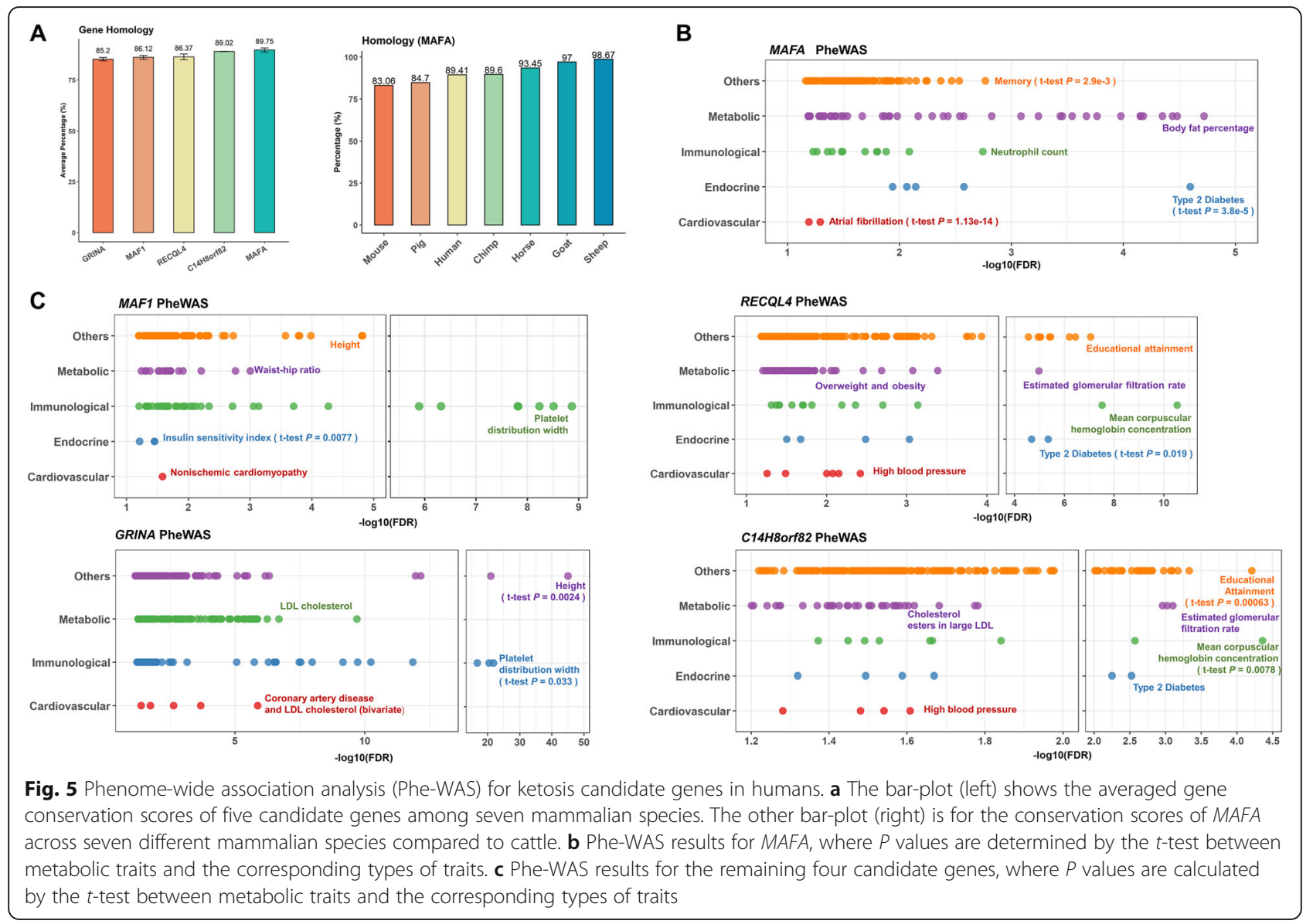

large enough. The DWGCNA approach constructs coexpression networks separately for different datasets to uncover the differences in modules [27, 33, 34].

We validated the detected candidate genes by using cross-species Phe-WAS analysis, which took advantage of rich resources in humans. These results highlight that the integrative analysis of multiple layers of biological data, including cross-species data, is promising to explore the underlying molecular mechanism of complex diseases and traits [15, 18, 35-37]. In this study, we used UMD3.1.1 as reference genome instead of the new assembly (ARS-USD 1.025), as our previous GWAS was conducted based on UMD3.1.1. However, future studies should use the new assembly.

Compared to ketosis, the plasma bio-indicators serve as intermediate phenotypes, which are more directly associated with alterations of gene expression induced by ketosis. The low calcium level in blood can cause ketosis and hypocalcemia, while ketosis leads to insulin resistance, thereby raising the risk of other metabolic diseases [38, 39]. Previous studies proposed that the function of HDL was to transport cholesterol from body tissues to the liver, serving as a "good" lipoprotein [40-42]. This was in line with our findings that the expression of several genes (e.g., C14H8orf82 and ACSS1), which had lower expression levels in the post-partum ketosis group compared to others, were positively correlated with HDL, leading to a lower HDL level in animals with post-partum ketosis (Fig. 3b).

Since gene expression is highly context-specific, it is thus important to choose the "right" tissue at the "right" physiological stages when studying the molecular mechanisms underlying a given trait $[18,43]$. For instance, in our study, we observed that gene co-expression modules, which were significantly correlated with post-partum ketosis rather than other status (e.g., pre-partum ketosis), were significantly enriched for GWAS signals of ketosis. This is consistent with findings in our previous study on mastitis, in which we found that the genetic variants of mastitis were specifically and significantly enriched in genes that were differentially expressed in liver at early time points (e.g., $3 \mathrm{~h}$ ) rather than at the late ones (e.g., $24 \mathrm{~h})$ post E. coli infection [18]. It is thus of great interest to collect more RNA-Seq data from multiple time points in the transition period to further explore the causal genes for ketosis in future studies.

In this study, we detected five candidate genes for ketosis, which showed high sequence conservation among 
mammals. By employing Phe-WAS of their human orthologues on a large range of complex traits and diseases in humans, we confirmed their key roles in metabolism and immunology. For instance, compared to other types of traits, MAFA showed significantly higher associations with metabolic and endocrine traits (e.g., Body fat percentage, FDR $=2.64 \mathrm{e}-05$; Type 2 Diabetes, $\mathrm{FDR}=1.9 \mathrm{e}-03) . M A F A$ is engaged in insulin secretion, glucose response, and transcriptional regulation [44, 45]. Previous studies reported that MAFA was a transcription factor binding RIPE3b, a conserved enhancer element that regulated pancreatic beta cell-specific expression of the insulin gene, which was involved in Insulinomatosis and Diabetes Mellitus $[44,46]$. Singh et al. also proposed that MAFA was implicated in the regulation of immunomodulatory cytokines such as interferon- $\beta$ (IFN $\beta 1$ ) [47]. In addition, glucose and lipid metabolic disorders are risk factors that can induce ketosis and immune-relevant diseases like mastitis. Therefore, we considered MAFA as a promising candidate gene for ketosis. However, it is required to validate this gene and other four candidate genes in future functional studies.

\section{Conclusions}

In this study, we integrated RNA-seq of blood leukocytes with large-scale GWAS results to detect genes/pathways underlying ketosis in cattle. Our results provide new insights into the molecular mechanism underlying ketosis, and highlights that the integrative analyses of omics data are promising for illustrating the genetic architecture underpinning complex traits and diseases.

\section{Methods}

\section{Animals and blood samples collection}

The experiment was conducted on the dairy farm of Beijing Sunlon Livestock Development Co Ltd. There were 2142 Holstein cows on the farm, of which a total of 78 lactation cows (parity 2-5) entered the dry period at the same time (that is, 2 months before the expected date). Their body condition scores and expected calving dates were similar. These cows were housed in the same pen and fed the same diet. The BHBA concentration in plasma was measured 2 weeks before calving and the fifth day after calving by using Optium ketone test strips with FreeStyle Precision blood ketone meter (Abbott Diabetes Care Ltd., IL, USA). Four cows with BHBA < $1.4 \mathrm{mmol} / \mathrm{L}$ on the fifth day after calving were selected as the healthy group; while 8 cows with BHBA $>1.4$ $\mathrm{mmol} / \mathrm{L}$ on the fifth day after calving were considered as ketosis syndrome group. The blood samples were collected from tail (coccygeal) veins of these animals using the following standard procedure. The animal was restrained properly, and its tail was raised vertically until it is horizontal to the ground. The venepuncture area was then disinfected with alcohol. On the midway along the body of a coccygeal vertebra, the needle was inserted perpendicularly to the surface of the skin to a depth of a few millimetres. The blood $(10 \mathrm{ml})$ was then collected using vacutainer tubes. Afterwards, the vacutainer tube was detached from the needle first, and the needle was then removed from the tail vein. The pressure with gauze was applied for 1 minute to ensure adequate hemostasis. The animals used in this study were released after blood sampling. Totally, 24 blood samples (two samples per animal i.e., prepartum and postpartum) were collected, followed by the immediate centrifugation for isolating plasma and leukocytes. Plasma samples were stored at $-20^{\circ} \mathrm{C}$ for measuring six blood bioindicators (e.g., total cholesterol, TC; total triglyceride, TG; high-density lipoprotein, HDL; low-density lipoprotein, LDL; calcium, Ca; and insulin, INS), while leukocytes were stored in the liquid nitrogen for further RNA sequencing. The bio-indicators of blood plasma were determined using commercial assay kits according to the manufacturer's methods (Laibotairui Technology Development Co Ltd., Beijing, China).

\section{mRNA extraction and sequencing}

We extracted total RNA from 24 blood leukocytes samples using TRIzol reagent (Invitrogen, Carlsbad, CA, USA). Then, we purified the RNA samples (AMPure XP system) and checked their quality using NanoPhotometer $^{\bullet}$ spectrophotometer (IMPLEN, CA, USA). We assessed RNA quality on the Agilent Bioanalyzer 2100 system. The concentration of the RNA samples ranged from 71.8 to $316 \mathrm{ng} / \mu \mathrm{l}$, and their RNA Integrity Numbers (RIN) were greater than six, which was enough for gene expression analysis [48-50]. We performed the preparation of cDNA library and the RNA sequencing in Novegene Co Ltd. (Beijing, China), generating pairedend reads at $150 \mathrm{bp}$ length (PE150) on the Illumina HiSeq 2500 platform. Ultimately, we obtained an average of 25 million read pairs per sample, which ranged from 21 million to 31 million across 24 samples.

\section{Bioinformatics analysis of RNA-seq}

We conducted quality control for raw reads using FastQC v0.11.3 (http://www.bioinformatics.babraham.ac.uk/projects/ fastqc/). We filtered adapters and reads with low quality, in which more than half of bases had quality $<=20$ or more than $10 \%$ of bases were missing (Ns $>10 \%$ ), to obtain clean reads. Quality equals to $-10 \times \log _{10}(e)$, where $e$ is error rate. We then used Hisat2 v2.0.5 (https://ccb.jhu.edu/software/ hisat2/) [51] aligner with default parameters (e.g., --n-ceil: L, 0, 0.15; -mp: $M X=6, M N=2$; np: 1; -rdg: 5, 3; -efg: 5, 3; -score-min: $\mathrm{L}, 0,-0.2)$ according to the software manual to map clean reads to the cattle reference genome assembly (UMD3.1.1) [52]. We downloaded the reference genome 
and all the corresponding annotation files from the Ensembl genome browser 94 (http://oct2018.archive.ensembl.org/ Bos_taurus/Info/Index). We used SAMtools v1.6.0 (http:// samtools.sourceforge.net/) [53] to transform alignment files from SAM format to sorted BAM format, and then employed the FeatureCounts v1.5.0 (http://bioinf.wehi.edu. $\mathrm{au} /$ featureCounts/) [54] to compute read counts for each of the 24,616 annotated Ensembl genes (defined as from transcriptional start site, TSS, to transcriptional end site, TES), including protein-coding genes $(n=19,968)$ and non-coding genes. All software were used with default parameters. For comparing gene expression across genes and samples, we normalized the raw read counts of genes using TPM method [55]. TPM is a normalization method to correct for gene length and sequencing depth to make genes comparable among samples. The raw read count of a gene was first divided by its length in kilobases (RPK), and then all RPK values of genes within a sample were summed up and divided by 1,000,000 to get "per million" scaling factor. The TPM of a gene was obtained through dividing its RPK value by the "per million" scaling factor. We kept genes with TPM (Transcripts Per Million) $>1$ and MAD (Median Absolute Deviation, measuring the variability of gene expression across samples) $>0.01$ (top $75 \%$ of MAD) for the subsequent analyses.

\section{Weighted gene correlation network analysis (WGCNA)}

We employed the WGCNA (v1.12.0), implemented in R, to construct gene co-expression network [56]. Briefly, as recommended by the WGCNA, we used the Variance Stabilizing Transformation (VST) procedure, implemented in DESeq2 [57], to normalize the matrix of read counts, yielding a matrix of values which was approximately homoskedastic (having constant variance along the range of mean values). We transformed this normalized matrix to a similarity matrix based on the pairwise Pearson's correlation among genes, and then converted the similarity matrix into an adjacency matrix. By using dynamic hybrid cutting method, we clustered genes with similar expression patterns $(r>0.9)$ into 16 distinct modules. We correlated (Pearson's correlation) eigengenes of 16 modules, defined as the first principle component of the corresponding expression matrix, with physiological states and seven plasma bio-indicators. The hub genes within significant modules were determined with connectivity greater than 30 and gene significance greater than 0.2 .

Single-maker GWAS and GWAS signal enrichment analysis We obtained summary statistics of single-marker GWAS $(n \approx 10,000)$ for ketosis and five health traits in cattle, which was described previously [10]. Livability as a composite trait is correlated with disease traits, reflecting a cow's overall ability to stay alive in a milking herd by measuring the percentage of on-farm deaths per lactation [10]. DSAB, highly associated with KETO, occurs when the abomasum fills with gas and moves from the floor to the top of the abdomen. CALC is a metabolic disorder with a low blood calcium level, while MAST is the inflammation of mammary gland. METR is the inflammation of uterus, and often occurs when a cow has a suppressed immune system after calving. Briefly, we considered de-regressed breeding values (predicted transmitting abilities - PTA) of Holstein bulls as phenotypes, while adjusted for all known co-variables, such as herd, year, season, and parity. We used imputed sequence markers $(n=2,619,418)$ with an averaged imputation accuracy of $96.7 \%$, minor allele frequency $(\mathrm{MAF})>0.01$ and Hardy-Weinberg Equilibrium (HWE) test $(P>10 \mathrm{e}-06)$ to conduct GWAS. We employed the following linear mixed model, implemented in MMAP software (https://mmap.github.io/), to test for association of genomic variants with phenotype:

$$
\mathbf{y}=\mu+\mathbf{X} b+\mathbf{g}+\mathbf{e}
$$

where $\mathbf{y}$ is phenotype, $\mu$ is the population mean, $\mathbf{X}$ is the genotype of a genomic marker (coded as 0,1 , or 2 ), $b$ is the marker effect, $\mathbf{g} \sim \mathrm{N}\left(\mathbf{0}, \sigma_{\mathrm{g}}^{2} \mathbf{G}\right)$ is the polygenic effect accounting for relationship, while $\mathbf{e} \sim \mathrm{N}\left(\mathbf{0}, \sigma_{\mathrm{e}}^{2} \mathbf{R}\right)$ is the residual. $\mathbf{G}$ is the genomic relationship matrix, built using $\mathrm{HD}$ markers with $\mathrm{MAF}>0.01$. $\mathbf{R}$ is a diagonal matrix with $R_{i i}=1 / r_{i}^{2}-1$, where $r_{i}^{2}$ is the reliability of phenotype for the $\mathrm{i}^{\text {th }}$ individual.

We applied a sum-based method, implemented in the $\mathrm{R}$ package for Quantitative Genetic and Genomic analyses (QGG package; http://psoerensen.github.io/qgg/) [58], for GWAS signal enrichment analyses across all 16 gene co-expression modules detected by WGCNA. The sum-based method uses signals of all markers within a pre-defined list of genes (i.e., a gene module), whereas the count-based method only uses signals of significant marker (e.g., $p<0.01$ ). Previous studies demonstrated that sum-based approach has equal or better power to detect genomic regions that are enriched with GWAS signals compared to other commonly used methods (e.g., count-based method), particularly in highly polygenic phenotypes [15, 18, 35-37]. Briefly, we calculated the following summary statistics for each gene coexpression module:

$$
T \text { sum }=\sum_{i=1}^{n} b^{2}
$$

where $T_{\text {sum }}$ is the summary statistics for a tested gene module, $b$ is the estimate of marker effect obtained in the single-marker GWAS, and $n$ is the number of SNPs located in the genes ( $20 \mathrm{~kb}$ up and downstream) within the module being tested. We determined the association of a module with a trait via a 10,000-times circular- 
genotype permutation test [58]. We calculated an empirical $P$ value for the module as the proportion of random $T_{\text {sum }}$ from permutation greater than the observed $T_{\text {sum }}$. The details were described previously [36]. In total, we analysed all 16 gene modules across six traits. We corrected raw $P$ values for the multiple testing using FDR method implemented in R (p.adjust function), and then considered FDR $<0.05$ as significant. Within significant modules, we considered genes (including $20 \mathrm{~kb}$ up and down-stream of genic regions, i.e., regulatory regions), whose top SNP showed $P<10 \mathrm{e}-05$ in GWAS, as candidate genes of ketosis. We used intersect function in BEDTools [59] to link SNP to its target gene.

\section{Other down-stream bioinformatics analyses}

To investigate functions of genes in our significant gene coexpression modules, we conducted functional enrichment analysis using the hypergeometric test based on Gene Ontology database and performed protein-protein interaction analysis using STRING v11 database with default settings (https://string-db.org/). We performed tissue and cell enrichment analysis for all 16 gene modules to detect tissues and cell types in which the corresponding genes were more likely to be highly and specifically expressed. Our previous study analyzed 732 RNA-Seq datasets, including 91 different tissue or cell types in cattle, using the same pipeline to detect genes with tissue or cell type-specific expression while accounting for known covariates. The details of the tissue/ cell type-specific genes were summarized previously [60] (http://cattlegeneatlas.roslin.ed.ac.uk/). Briefly, for each gene in a given tissue, we computed a $t$-statistics to measure its tissue specificity of expression by fitting a linear regression model (samples of the target tissue were coded as 1 , while the remaining ones as -1 ). We then ranked genes based on their $t$-statistics (higher value, higher tissue-specificity) in each tissue, and chose the top $5 \%$ of genes as tissue-specific genes $[43,60]$. We employed the hypergeometric test, similar to the GO enrichment analysis, to detect the enriched tissues and cell types for a given gene module. We adjusted $P$ values for multiple testing using the FDR method [61]. We used the BLAST function provided by NCBI (https:// blast.ncbi.nlm.nih.gov/Blast.cgi) to obtain the sequence similarities between candidate genes in cattle and their orthologues among other mammalian species. To test whether human orthologues of ketosis-candidate genes are associated with similar traits in humans. We performed the Phe-WAS analysis for human orthologues of these genes across 3302 human phenotypes (https://atlas.ctglab.nl/). Phenome-wide association analysis (Phe-WAS) is a study design that tests associations of a given SNP or gene with a large number of different phenotypes, which is a complementary approach to GWAS. It has been proved useful in recovering previously detected genotype-phenotype associations and in discovering new ones $[62,63]$.

\section{Supplementary information}

Supplementary information accompanies this paper at https://doi.org/10. 1186/s12864-020-06909-z.

Additional file 1: Table S1. Summary of RNA-Seq data mapping information.

Additional file 2: Table S2. Summary of seven blood bio-indicators across 24 samples.

Additional file 3: Table S3. Gene Ontology (GO) enrichment analysis of five gene modules.

Additional file 4: Table S4. Tissue enrichment analysis of all gene modules.

Additional file $\mathbf{5}$ Table S5. Connectivity, significance and membership of genes in modules.

Additional file 6: Figure S1. Correlation between GWAS enrichments of ketosis and module-states associations from WGCNA.

Additional file 7: Table S6. Phenome-wide analysis results for five candidate genes.

\section{Abbreviations}

RNA-Seq: RNA Sequencing; WGCNA: Weighted gene co-expression network analysis; GWAS: Genome wide association studies; Phe-WAS: Phenome-wide association analysis; TC: Total cholesterol; TG: Total triglyceride; HDL: Highdensity lipoprotein; LDL: Low-density lipoprotein; Ca: Calcium; INS: Insulin; BHBA: Beta-hydroxybutyrate; NEB: Negative energy balance; DMI: Dry material intake; KETO: Ketosis; MAST: Mastitis; DSAB: Displaced abomasum; METR: Metritis; CALC: Hypocalcemia; BTA: Bos taurus autosome; MAD: Median absolute deviation; GO: Gene ontology; QTL: Quantitative trait loci;

SNP: Single nucleotide polymorphism; RIN: RNA integrity number; FDR: False discovery rate; TPM: Transcripts per kilobase million; TSS: Transcriptional start site; TES: Transcriptional end site; RPK: Reads per kliobase; VST: Variance Stabilizing Transformation; PTA: Predicted transmitting abilities; MAF: Minor allele frequency; HWE: Hardy-Weinberg Equilibrium; DWGCNA: Differential weighted gene co-expressed network analysis

\section{Acknowledgements}

We thank Yan Wang (Jinyindao Dariy Farm) for her technical assistance collecting samples, Yajing Wang (China Agricultural University) for her valuable comments on the manuscript.

\section{Authors' contributions}

$L M, L F$ and $Y Z$ conceived and designed the experiments. $Z Y, H H, D D, J S, C M$, $J C$ and GG collected samples and/or generated data. ZY, HH, EF, DS, DD, JS, $\mathrm{CM}, J C$ and GG and LF performed computational and statistical analyses. $Z Y$, $L F$, EF, GEL, LM and YZ wrote the manuscript. All authors read, revised and approved the final manuscript.

\section{Funding}

This work was supported by the China Agricultural Research System (CARS37), National Key Research and Development Project (2019YFE0106800), and Beijing Dairy Industry Innovation Team Fund (BAIC06). The funders played no role in study design; in the collection, analysis, and interpretation of data; in the writing of the manuscript; and in the decision to submit the manuscript for publication.

\section{Availability of data and materials}

All 24 high-throughput RNA sequencing data analyzed in this study are deposited in Sequence Read Archive (SRA) database in NCBI under accession number PRJNA605719 (https://www.ncbi.nlm.nih.gov/bioproject/PRJNA60571 9). The reference genome (ftp://ftp.ensembl.org/pub/release-94/fasta/bos_ taurus/dna/Bos_taurus.UMD3.1.dna.toplevel.fa.gz) and gene annotation files (ftp://ftp.ensembl.org/pub/release-94/gtf/bos_taurus/Bos_taurus.UMD3.1.94. gtf.gz) of bovine UMD3.1.1 were downloaded from Ensembl v94 (https:// www.ensembl.org/index.html). 


\section{Ethics approval and consent to participate}

We carried out the entire sample collection procedures in strict accordance with the protocol approved by the Animal Welfare Committee of China Agricultural University.

\section{Consent for publication}

Not applicable.

\section{Competing interests}

The authors declare that they have no competing interests, except that George E. Liu is a member of the editorial board (Associate Editor) of the BMC Genomics journal.

\section{Author details}

${ }^{1}$ National Engineering Laboratory for Animal Breeding, Key Laboratory of Animal Genetics, Breeding and Reproduction of Ministry of Agriculture and Rural Affairs, College of Animal Science and Technology, China Agricultural University, Beijing 100193, China. ${ }^{2}$ College of Animal Science and Veterinary Medicine, Henan Agricultural University, Zhengzhou 450002, China. ${ }^{3}$ Department of Animal and Avian Sciences, University of Maryland, College Park, MD 20742, USA. ${ }^{4}$ Department of Clinical Veterinary Medicine, College of Veterinary Medicine, China Agricultural University, Beijing 100193, China. ${ }^{5}$ Beijing Sunlon Livestock Development Co Ltd., Beijing 100076, China. ${ }^{6}$ Animal Genomics and Improvement Laboratory, Henry A. Wallace Beltsville Agricultural Research Center, Agricultural Research Service, Agricultural Research Service, USDA, Beltsville, MD 20705, USA. ${ }^{7}$ MRC Human Genetics Unit at the Institute of Genetics and Molecular Medicine, University of Edinburgh, Edinburgh EH4 2XU, UK.

Received: 19 February 2020 Accepted: 13 July 2020

Published online: 17 July 2020

\section{References}

1. Esposito G, Irons PC, Webb EC, Chapwanya A. Interactions between negative energy balance, metabolic diseases, uterine health and immune response in transition dairy cows. Anim Reprod Sci. 2014;44:60-71.

2. Overton TR, McArt JAA, Nydam DV. A 100-year review: metabolic health indicators and management of dairy cattle. J Dairy Sci. 2017; 100(12):10398-417.

3. Liang D, Arnold LM, Stowe CJ, Harmon RJ, Bewley JM. Estimating US dairy clinical disease costs with a stochastic simulation model. J Dairy Sci. 2017; 100(2):1472-86.

4. Xu S, Wu Z, Du W, Li S, Cao Z. Evaluation of economic loss of dairy cow ketosis. China Dairy Cattle. 2014;15:62-4

5. Stengärde L, Holtenius K, Emanuelson U, Hultgren J, Niskanen R, Tråvén M. Blood parameters in Swedish dairy herds with high or low incidence of displaced abomasum or ketosis. Vet J. 2011;190:124-30.

6. Oetzel GR. Monitoring and testing dairy herds for metabolic disease. Vet Clin North Am Food Anim Pract. 2004;20:651-74.

7. LeBlanc SJ, Leslie KE, Duffield TF. Metabolic predictors of displaced abomasum in dairy cattle. J Dairy Sci. 2005;88:159-70

8. Seifi HA, LeBlanc SJ, Leslie KE, Duffield TF. Metabolic predictors of postpartum disease and culling risk in dairy cattle. Vet J. 2011;188:216-20.

9. Mostert PF, Bokkers EAM, van Middelaar CE, Hogeveen $\mathrm{H}$, de Boer IJM. Estimating the economic impact of subclinical ketosis in dairy cattle using a dynamic stochastic simulation model. Animal. 2018;12:145-54.

10. Freebern E, Santos DJA, Fang L, Jiang J, Parker Gaddis KL, Liu GE, VanRaden PM, Maltecca C, Cole JB, Ma L. GWAS and fine-mapping of livability and six disease traits in Holstein cattle. BMC Genomics. 2020;21:41.

11. Uribe HA, Kennedy BW, Martin SW, Kelton DF. Genetic parameters for common health disorders of Holstein cows. J Dairy Sci. 1995;78:421-30.

12. Kadarmideen HN, Thompson R, Simm G. Linear and threshold model genetic parameters for disease, fertility and milk production in dairy cattle. Animal Sci. 2000;71:411-9.

13. Heringstad B, Chang YM, Gianola D, Klemetsdal G. Genetic analysis of clinical mastitis, milk fever, ketosis, and retained placenta in three lactations of Norwegian red cows. J Dairy Sci. 2005;88:3273-81.

14. Edwards SM, Sørensen IF, Sarup P, Mackay TF, Sørensen P. Genomic prediction for quantitative traits is improved by mapping variants to gene ontology categories in Drosophila melanogaster. Genetics. 2016; 203:1871-83.
15. Fang L, Sahana G, Ma P, Su G, Yu Y, Zhang S, Lund MS, Sørensen P. Exploring the genetic architecture and improving genomic prediction accuracy for mastitis and milk production traits in dairy cattle by mapping variants to hepatic transcriptomic regions responsive to intra-mammary infection. Genet Sel Evol. 2017;49:44.

16. Fang L, Sahana G, Ma P, Su G, Yu Y, Zhang S, Lund MS, Sørensen P. Use of biological priors enhances understanding of genetic architecture and genomic prediction of complex traits within and between dairy cattle breeds. BMC Genomics. 2017;18:604.

17. Fang L, Liu S, Liu M, Kang X, Shudai L, Li B, Connor E, Baldwin R, Tenesa A, Liu G, et al. Functional annotation of the cattle genome through systematic discovery and characterization of chromatin states and butyrate-induced variations. BMC Biol. 2019:17:68

18. Fang L, Sahana G, Su G, Yu Y, Zhang S, Lund MS, Sorensen P. Integrating sequence-based GWAS and RNA-Seq provides novel insights into the genetic basis of mastitis and milk production in dairy cattle. Sci Rep. 2017;7: 45560.

19. McCabe M, Waters S, Morris D, Kenny D, Lynn D, Creevey C. RNA-seq analysis of differential gene expression in liver from lactating dairy cows divergent in negative energy balance. BMC Genomics. 2012;13:193.

20. Stilling RM, van de Wouw M, Clarke G, Stanton C, Dinan TG, Cryan JF. The neuropharmacology of butyrate: the bread and butter of the microbiotagut-brain axis? Neurochem Int. 2016;99:110-32.

21. Wiley NC, Dinan TG, Ross RP, Stanton C, Clarke G, Cryan JF. The microbiota-gut-brain axis as a key regulator of neural function and the stress response: implications for human and animal health. J Dairy Sci. 2017:95:3225-46.

22. Carabotti M, Scirocco A, Maselli MA, Severi C. The gut-brain axis: interactions between enteric microbiota, central and enteric nervous systems. Ann Gastroenterol. 2016;29:240.

23. Joscelyn J, Kasper LH. Digesting the emerging role for the gut microbiome in central nervous system. Mult Scler. 2014;20:1553-9.

24. Singh DK, Karmakar P, Aamann M, Schurman SH, May A, Croteau DL, Burks L, Plon SE, Bohr VA. The involvement of human RECQL4 in DNA doublestrand break repair. Aging Cell. 2010;9:358-71.

25. Sangrithi MN, Bernal JA, Madine M, Philpott A, Lee J, Dunphy WG, Venkitaraman AR. Initiation of DNA replication requires the RECQL4 protein mutated in Rothmund-Thomson syndrome. Cell. 2005;121:887-98.

26. Hu L, Smith TF, Goldberger G. LFG. A candidate apoptosis regulatory gene family. Apoptosis. 2009;14:1255-65.

27. van Dam S, Võsa U, van der Graaf A, Franke L, de Magalhães JP. Gene coexpression analysis for functional classification and gene-disease predictions. Brief Bioinform. 2018;19(4):575-92.

28. Gerring ZF, Gamazon ER, Derks EM. Major depressive disorder working Group of the Psychiatric Genomics C. A gene co-expression network-based analysis of multiple brain tissues reveals novel genes and molecular pathways underlying major depression. PLoS Genet. 2019;15:e1008245.

29. Qiu J, Du Z, Wang Y, Zhou Y, Zhang Y, Xie Y, LV Q. Weighted gene coexpression network analysis reveals modules and hub genes associated with the development of breast cancer. Medicine (Baltimore). 2019;98: e14345-e14345.

30. Li J, Zhou D, Qiu W, Shi Y, Yang J-J, Chen S, Wang Q, Pan H. Application of weighted gene co-expression network Analysis for data from paired design. Sci Rep. 2018;8:622.

31. Sabino M, Carmelo VAO, Mazzoni G, Cappelli K, Capomaccio S, AjmoneMarsan P, Verini-Supplizi A, Trabalza-Marinucci M, Kadarmideen HN. Gene co-expression networks in liver and muscle transcriptome reveal sex-specific gene expression in lambs fed with a mix of essential oils. BMC Genomics. 2018:19:236-6.

32. Fonseca PA-O, Suárez-Vega A, Cánovas AA-O. Weighted Gene Correlation Network Meta-Analysis Reveals Functional Candidate Genes Associated with High- and Sub-Fertile Reproductive Performance in Beef Cattle. Genes (Basel). 2020;11:543.

33. Fuller TF, Ghazalpour A, Aten JE, Drake TA, Lusis AJ, Horvath S. Weighted gene coexpression network analysis strategies applied to mouse weight. Mamm Genome. 2007;18(6-7):463-72.

34. Fuller T., Langfelder P., Presson A., Horvath S. (2011) Review of weighted gene Coexpression network Analysis. In: Lu HS., Schölkopf B., Zhao H. (eds) Handbook of statistical bioinformatics. Springer Handbooks of Computational Statistics. Springer, Berlin, Heidelberg. 
35. Fang L, Sørensen P, Sahana G, Panitz F, Su G, Zhang S, Yu Y, Li B, Ma L, Liu $\mathrm{G}$, et al. MicroRNA-guided prioritization of genome-wide association signals reveals the importance of microRNA-target gene networks for complex traits in cattle. Sci Rep. 2018;8:9345.

36. Rohde PD, Demontis D, Cuyabano BCD, Børglum AD, Sørensen P. Covariance association test (CVAT) identifies genetic markers associated with schizophrenia in functionally associated biological processes. Genetics. 2016:203:1901

37. Sørensen IF, Edwards SM, Rohde PD, Sørensen P. Multiple trait covariance association test identifies gene ontology categories associated with chill coma recovery time in Drosophila melanogaster. Sci Rep. 2017;7:2413.

38. Hayirli A. The role of exogenous insulin in the complex of hepatic lipidosis and ketosis associated with insulin resistance phenomenon in postpartum dairy cattle. Vet Res Commun. 2006;30(7):749-74.

39. Veech RL. The therapeutic implications of ketone bodies: the effects of ketone bodies in pathological conditions: ketosis, ketogenic diet, redox states, insulin resistance, and mitochondrial metabolism. Prostaglandins Leukot Essent Fatty Acids. 2004;70(3):309-19.

40. Khera AV, Cuchel M, de la Llera-Moya M, Rodrigues A, Burke MF, Jafri K, French BC, Phillips JA, Mucksavage ML, Wilensky RL, et al. Cholesterol efflux capacity, high-density lipoprotein function, and atherosclerosis. N Engl J Med. 2011;364(2):127-35.

41. Ansell BJ, Watson KE, Fogelman AM, Navab M, Fonarow GC. High-density lipoprotein function recent advances. J Am Coll Cardiol. 2005;46:1792.

42. Fisher EA, Feig JE, Hewing B, Hazen SL, Smith JD. High-density lipoprotein function, dysfunction, and reverse cholesterol transport. Arterioscler Thromb Vasc Biol. 2012;32:2813-20.

43. Finucane HK, Reshef YA, Anttila V, Slowikowski K, Gusev A, Byrnes A, Gazal S, Loh PR, Lareau C, Shoresh N, et al. Heritability enrichment of specifically expressed genes identifies disease-relevant tissues and cell types. Nat Genet. 2018;50:621-9.

44. Zhang C, Moriguchi T, Kajihara M, Esaki R, Harada A, Shimohata H, Oishi H, Hamada M, Morito N, Hasegawa K, et al. MafA is a key regulator of glucosestimulated insulin secretion. Mol Cell Biol. 2005;25:4969.

45. Wang H, Brun T, Kataoka K, Sharma AJ, Wollheim CB. MAFA controls genes implicated in insulin biosynthesis and secretion. Diabetologia. 2007;50:348-58.

46. Olbrot M, Rud J, Moss LG, Sharma A. Identification of beta-cell-specific insulin gene transcription factor RIPE3b1 as mammalian MafA. Proc Natl Acad Sci. 2002;99:6737-42.

47. Singh T, Sarmiento L, Luan C, Prasad RB, Johansson J, Cataldo LR, Renstrom E, Soneji S, Cilio C, Artner I. MafA expression preserves immune homeostasis in human and mouse islets. Genes. 2018;9:644.

48. Romero GI, Pai AA, Tung J, Gilad Y. RNA-seq: impact of RNA degradation on transcript quantification. BMC Biol. 2014;12:42.

49. Davila JI, Fadra NM, Wang X, McDonald AM, Nair AA, Crusan BR, Wu X Blommel JH, Jen J, Rumilla KM, et al. Impact of RNA degradation on fusion detection by RNA-seq. BMC Genomics. 2016;17:814.

50. Cánovas A, Rincón G, Bevilacqua C, Islas-Trejo A, Brenaut P, Hovey RC, Boutinaud M, Morgenthaler C, MK VK, Martin P, et al. Comparison of five different RNA sources to examine the lactating bovine mammary gland transcriptome using RNA-sequencing. Sci Rep. 2014;4:5297.

51. Kim D, Langmead B, Salzberg SL. HISAT: a fast spliced aligner with low memory requirements. Nat Methods. 2015;12(4):357-60.

52. Bovine Genome S, Analysis C, Elsik CG, Tellam RL, Worley KC, Gibbs RA, Muzny DM, Weinstock GM, Adelson DL, Eichler EE, et al. The genome sequence of taurine cattle: a window to ruminant biology and evolution. Science. 2009;324(5926):522-8.

53. Li H, Handsaker B, Wysoker A, Fennell T, Ruan J, Homer N, Marth G, Abecasis G, Durbin R. 1000 Genome project data processing subgroup: the sequence alignment/map format and SAMtools. Bioinformatics. 2009;25(16):2078-9.

54. Liao Y, Smyth GK, Shi W. FeatureCounts: an efficient general purpose program for assigning sequence reads to genomic features. Bioinformatics. 2014:30(7):923-30

55. Conesa A, Madrigal P, Tarazona S, Gomez-Cabrero D, Cervera A, McPherson A, Szcześniak MW, Gaffney DJ, Elo LL, Zhang X, et al. A survey of best practices for RNA-seq data analysis. Genome Biol. 2016;17:13.

56. Langfelder P, Horvath S. WGCNA. an R package for weighted correlation network analysis. BMC Bioinformatics. 2008:9:559.

57. Love Ml, Huber W, Anders S. Moderated estimation of fold change and dispersion for RNA-seq data with DESeq2. Genome Biol. 2014;15(12):550.
58. Rohde PD, Sorensen IF, Sorensen P. qgg: an R package for large-scale quantitative genetic analyses. Bioinformatics. 2019:btz955. https://doi.org/10. 1093/bioinformatics/btz955.

59. Quinlan AR, Hall IM. BEDTools: a flexible suite of utilities for comparing genomic features. Bioinformatics. 2010;26(6):841-2.

60. Fang L, Cai W, Liu S, Canela-Xandri O, Gao Y, Jiang J, Rawlik K, Li B, Schroeder SG, Rosen BD, et al. Comprehensive analyses of 723 transcriptomes enhance genetic and biological interpretations for complex traits in cattle. Genome Res. 2020;30:790-801.

61. Benjamini Y, Drai D, Elmer G, Kafkafi N, Golani I. Controlling the false discovery rate in behavior genetics research. Behav Brain Res. 2001;125:279-84.

62. Hebbring SJ. The challenges, advantages and future of phenome-wide association studies. Immunology. 2014;141:157-65.

63. Cronin RM, Field JR, Bradford Y, Shaffer CM, Carroll RJ, Mosley JD, Bastarache L, Edwards TL, Hebbring SJ, Lin S, et al. Phenome-wide association studies demonstrating pleiotropy of genetic variants within FTO with and without adjustment for body mass index. Front Genet. 2014;5:250.

\section{Publisher's Note}

Springer Nature remains neutral with regard to jurisdictional claims in published maps and institutional affiliations.
Ready to submit your research? Choose BMC and benefit from:

- fast, convenient online submission

- thorough peer review by experienced researchers in your field

- rapid publication on acceptance

- support for research data, including large and complex data types

- gold Open Access which fosters wider collaboration and increased citations

- maximum visibility for your research: over $100 \mathrm{M}$ website views per year

At BMC, research is always in progress.

Learn more biomedcentral.com/submissions 